\title{
Percepção do bem-estar e da qualidade de vida em acadêmicos do curso de Educação Física
}

\author{
Perception of the welfare and quality of life in stroke scholars of \\ Physical Education
}

Francisco Edson da Silva Ferreira Filho', Romário Pinheiro Lustosa², Thiago Medeiros da Costa Daniele Ariane Teixeira dos Santos ${ }^{4}$, Natalia Macedo Uchoa ${ }^{5}$, Francisco Nataniel Macedo Uchoa ${ }^{1,6}$

'Faculdade Integrada da Grande Fortaleza (FGF), Fortaleza, CE, Brasil.

2Centro Universitário Estácio do Ceará, Fortaleza, CE, Brasil.

${ }^{3}$ Universidade Federal do Ceará - UFC, Fortaleza, CE, Brasil.

${ }^{4}$ Universidade Federal do Rio Grande do Norte - UFRN, Natal, RN, Brasil.

${ }^{5}$ Universidade de Fortaleza - UNIFOR, Fortaleza, CE, Brasil.

Eniversidade de Trás-0s-Montes e Alto Douro, Vila Real, Portugal.

Recebido em: 23/03/2016 / Aceito em: 15/04/2016

romario-lustosa@hotmail.com

\section{RESUMO}

Objetivo: o presente estudo teve como objetivo analisar o perfil de estilo de vida de acadêmicos do curso de Licenciatura em Educação Física em uma Instituição particular de ensino superior. Método: foram avaliados 100 acadêmicos de ambos os sexos, através do questionário Pentáculo do Bem-Estar. Resultados e discussão: pôde ser constatada a presença de afirmações que declararam comportamentos com predominância de índices positivos em todos os itens, muito embora, também tenham sido encontrados alguns índices considerados regulares e para o resultado positivo do grupo, não prevaleceu em nenhum componente o índice negativo. Portanto, sugere-se que o perfil do estilo de vida dos acadêmicos investigados está, mesmo com poucas ressalvas, referente há alguns poucos índices regulares, em conformidade com o que preconiza a literatura, já que obtiveram a prevalência de índices positivos em todos os componentes. Considerações finais: diante do que foi tratado neste trabalho, sugere-se ao público alvo que adote meios para estarem sempre em busca do índice de estilo de vida positivo, assim como, a Instituição onde foi realizada a pesquisa, a realização de ações de conscientização e de incentivo para uma melhora do estilo de vida de seus acadêmicos.

Palavras-chave: Perfil de acadêmicos da educação física; Qualidade de vida; Saúde.

\section{ABSTRACT}

Objective: this study aimed analyze the lifestyle profile of Physical Education of the of academics in a particular institution of higher education. Methods: it was evaluated 100 students. Quality of life and its components was evaluated by the pentacle of Wellness. Results and discussion: it was observed the presence of statements that declared behavior with a predominance of positive results in all components, however, have also been found some regular and the positive outcome of the group, did not prevail any component negative index. Final considerations: therefore, it is suggested that lifestyle profile of the investigated academic, being even with few exceptions, referring there are a few regular indices, in accordance with which calls the literature, since it obtained the prevalence of positive results in all components, conducting awareness campaigns and incentive to an improvement in the lifestyle of their academics.

Keywords: Academic profile of physical education; Quality of university life; Health.

\section{INTRODUÇÃO}

A Organização Mundial da Saúde (OMS) estipula a adequação de padrões ideais para uma boa saúde; caracterizada como um estado de bem-estar social, físico 
e mental, sendo entendida como um recurso diário e não como objetivo de vida. ${ }^{1}$

A saúde é considerada como uma condição humana a diversos fatores e dimensões físicas, psicológicas e sociais. Nesse contexto, a saúde é entendida não apenas como a ausência de doenças, mas, como um completo bem-estar que envolve outras dimensões, além da biológica; outras evidências apontam para um entendimento da saúde como uma inter-relação com a qualidade de vida. ${ }^{2}$

A qualidade de vida, por sua vez, pode ser entendida como sensação íntima de conforto, bem-estar ou felicidade no desempenho de funções físicas, intelectuais e psíquicas dentro da realidade da família, do trabalho e dos valores da comunidade à qual pertence. Desta forma, qualidade de vida é o conjunto multifatorial adquirido ao longo da vida, que um indivíduo se apropria e vivencia para o seu bem-estar, nas diversas relações em que o mesmo se encontra inserido. ${ }^{3}$

Herdeiro da contemporaneidade e um dos principais males sociais, o sedentarismo desenvolveu-se com mais ênfase a partir da evolução tecnológica e propiciou aspectos severos da inatividade física, substituindo diversas atividades, por uma economia de esforços. O sedentarismo ou inatividade física é entendido pela OMS como o principal inimigo da saúde pública, sendo o quarto maior fator de risco para doenças crônicas, associado a mais de dois milhões de mortes ao ano em todo o mundo e de $75 \%$ dos casos de óbitos nas Américas. ${ }^{4}$

O sedentarismo é entendido como a falta ou a diminuição de alguma atividade física, que afeta a saúde, elemento fundamental do bem maior que um indivíduo tem na vida. Tendo em vista que o sedentarismo é um grande causador de mortes, bem como de inúmeras doenças, devemos, através de um estilo de vida saudável, adotar medidas que o afastem de nossas vidas. ${ }^{5}$

O estilo de vida é definido como um conjunto de ações cotidianas, que refletem as atitudes e valores das pessoas. Este conjunto de ações, que regem a vidas das pessoas, pode ou não, ao longo do tempo, mudar em função de alguns fatores que possam mostrar que as ações de hoje, não condizem mais com o momento. ${ }^{6}$

Complementando a definição anterior, outro autor adiciona que, o estilo de vida é caracterizado por hábitos em que os indivíduos apreendem e adotam durante suas vidas, em que se relacionam com a realidade familiar, ambiental e social. Após o entendimento do que foi exposto, podemos entender que o estilo de vida que uma pessoa utiliza é o principal meio de se chegar ao seu estado de saúde positivo ou negativo, portanto se uma pessoa adota um estilo de vida saudável, ela conserva sua saúde, no contrário, ela poderá fragilizar sua saúde. ${ }^{7}$

Dentro deste mesmo entendimento, sabemos que existem componentes que servem de referência para a análise do estilo de vida de cada pessoa como a nutrição, a atividade física, o relacionamento social, o comportamento preventivo e o controle de estresse, conforme preconiza. ${ }^{8}$

Atividade física é definida como estar fisicamente ativo até haver um aumento significativo no consumo de energia durante o trabalho, nas atividades sistemáticas da vida diária, ou até mesmo por momentos de lazer. ${ }^{3}$ Aprofundando um pouco mais esta definição, encontramos que, a atividade física, num sentido mais restrito, é todo movimento corporal, produzido por músculos esqueléticos, que provoca um gasto de energia. ${ }^{9}$ Então chegamos ao entendimento, que a atividade física é fundamental para um estilo de vida positivo, pois seus benefícios além de afastar o sedentarismo, contribuem significativamente em outros pontos para uma boa qualidade de vida.

$\mathrm{O}$ presente estudo tem como objetivo analisar o estilo de vida dos acadêmicos do curso de Licenciatura em Educação Física, já os específicos são: identificar fatores de estilo de vida associados aos padrões nutricionais, a atividade física, ao relacionamento social, ao controle de estresse e ao comportamento preventivo.

\section{MÉTODO}

O presente estudo caracteriza-se por uma análise descritiva, tendo como objetivo principal observar, registrar e analisar fenômenos, contudo, sem interferir ou influenciar. Este estudo objetiva traçar o perfil do estilo de vida de acadêmicos do curso de Licenciatura em Educação Física, em uma Instituição particular de Ensino Superior na cidade de Fortaleza. Foram selecionados 100 acadêmicos com idades entre 17 e 48 anos, do gênero masculino (64 alunos) e do gênero feminino (36 alunas). Todos os alunos aceitaram em participar do estudo e assinaram o Termo de consentimento livre esclarecido. Foram excluídos aqueles que não aceitaram em participar ou em assinar o Termo de Consentimento do estudo.

Para a análise dos dados, foi utilizado o questionário Pentáculo do Bem-Estar- Base Conceitual para Avaliação do Estilo de Vida de Indivíduos e Grupos. ${ }^{8}$ Este instrumento possui cinco componentes relacionados com o estilo de vida: nutrição, atividade física, relacionamento social, comportamento preventivo e controle de estresse. Estes cinco componentes são compostos por três questões cada. As questões foram respondidas de acordo com as seguintes alternativas:

(0) Zero - Absolutamente não faz parte do seu estilo de vida;

(1) Um - Raramente corresponde ao seu comportamento;

(2) Dois - Quase sempre verdadeiro no seu comportamento;

(3) Três - A afirmação é sempre verdadeira no seu dia-a-dia, faz parte do seu estilo de vida.

Estas respostas foram pontuadas de $(0)$ zero a (3) três pontos, como o exposto acima, em que cada componente do Pentáculo do Bem-Estar poderá somar (9) nove pontos e um total de (45) quarenta e cinco pontos na somatória dos cinco componentes por cada sujeito entrevistado.

Para cada questão temos abaixo os seguintes escores:

Menos de (1) um - índice negativo;

Entre (1) um e $(1,99)$ um vírgula noventa e nove índice regular;

Entre (2) dois e (3) três - índice positivo.

Os dados foram tratados, inicialmente através de métodos descritivos na pesquisa. Foram calculadas as médias dos componentes para identificar a porcen- 
tagem dos resultados obtidos. Para a representação pictural do Pentáculo do Bem-Estar em grupo, foi calculada a média das afirmativas dos entrevistados, sendo que para chegar ao número foi necessário multiplicar a frequência das respostas dos entrevistados pelo valor do peso de cada afirmativa, dividindo o total de cada item dos componentes encontrados pelo número de entrevistados.

\section{RESULTADO E DISCUSSÃO}

Os dados deste estudo foram divididos pelas características de cada componente do Pentáculo do Bem-Estar com suas respectivas variáveis. ${ }^{8}$

Conforme pode ser observado no componente nutrição, os resultados se destacam como: a) (3\%) dos alunos não incluem 5 porções de frutas/verduras em seu cotidiano, tendo assim um índice negativo. Outros $(43 \%)$ dos alunos registraram índice regular, pois relataram que raramente incluem 5 porções de frutas/ verduras em seu cotidiano. Já os restantes dos alunos apresentaram índice positivo, pois incluem, sempre ou quase sempre, 5 porções de frutas/verduras em seu cotidiano. Tanto os entrevistados que responderam sempre e quase sempre podem ser classificados em índice positivo de estilo de vida, pois as frutas e os vegetais fazem parte de uma rica e variada dieta com os nutrientes essenciais ao organismo humano; ${ }^{10}$

b) no segundo item ( $8 \%$ ) dos entrevistados, não evitam alimentos gordurosos e doces, em seu cotidiano, registrando assim índice negativo, enquanto (22\%) dos acadêmicos apresentam índice regular, pois raramente evitam estes alimentos gordurosos e doces. Os outros $(70 \%)$ se encontram com índice positivo, pois afirmaram que sempre ou quase sempre evitam estes alimentos. Pessoas que, incluem em sua alimentação diária o consumo de nutrientes com excesso de açúcar como refrigerantes e alimentos com alto teor de gordura, podem se tornarem obesas ou com excesso de peso. ${ }^{11}$ Segundo Lopes, ${ }^{3}$ com uma dieta baseada em qualidade e quantidade correta, o organismo ganha energia suficiente para desempenhar as funções que nosso corpo precisa. É necessário introduzir em nossa alimentação uma grande variedade de alimentos nas refeições diárias, já que nenhum alimento é disposto de todos os nutrientes que necessitamos. ${ }^{10}$ Podemos entender que os benefícios de uma boa alimentação, com grande variedade de alimentos, são bastante essenciais para os propósitos de um estilo de vida positivo e consequentemente de uma boa saúde.

c) no terceiro item, foi observado que nenhum dos entrevistados apresentou índices negativos, contudo $19 \%$ demonstraram valores (4 a 5 refeições ao dia).

No componente atividade física foi observado o seguinte: a) no primeiro item podemos observar que: $2 \%$ dos alunos apresentaram índice negativo, 18\% foram classificados como regular e o restante tiveram índice positivo, por responderem que sempre ou quase sempre

Tabela 1 - Aspectos gerais do estilo de vida e bem-estar individual.

\begin{tabular}{|c|c|c|c|c|c|}
\hline & Especificações & Nunca & Às vezes & Quase sempre & Sempre \\
\hline \multirow{3}{*}{ 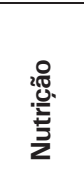 } & Alimentação diária inclui pelo menos 5 porções de frutas e hortaliças & $3 \%$ & $43 \%$ & $43 \%$ & $11 \%$ \\
\hline & Evita ingerir alimentos gordurosos e doces & $8 \%$ & $22 \%$ & $21 \%$ & $49 \%$ \\
\hline & Faz 4 a 5 refeições variadas ao dia, incluindo café da manhã completo & $0 \%$ & $19 \%$ & $40 \%$ & $41 \%$ \\
\hline \multirow{3}{*}{$\begin{array}{l}\frac{80}{0} \\
\frac{0}{0} \\
\frac{0}{0} \\
\frac{0}{0} \\
\frac{\pi}{0} \\
\frac{0}{4}\end{array}$} & $\begin{array}{l}\text { Realiza ao menos } 30 \text { min. de atividades físicas moderadas/intensas, de forma } \\
\text { contínua ou acumulada, } 5 \text { ou mais dias na semana }\end{array}$ & $2 \%$ & $17 \%$ & $33 \%$ & $48 \%$ \\
\hline & $\begin{array}{l}\text { Ao menos } 3 \text { vezes por semana realiza exercícios que envolvam força e } \\
\text { alongamento muscular }\end{array}$ & $2 \%$ & $18 \%$ & $19 \%$ & $51 \%$ \\
\hline & $\begin{array}{l}\text { No dia-a-dia, caminha ou pedala como meio de transporte e, preferencial- } \\
\text { mente, usa as escadas ao invés o elevador }\end{array}$ & $6 \%$ & $30 \%$ & $34 \%$ & $30 \%$ \\
\hline \multirow{3}{*}{ 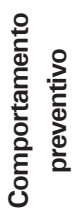 } & Conhece pressão arterial e nível de colesterol e procura controlá-los & $13 \%$ & $27 \%$ & $28 \%$ & $32 \%$ \\
\hline & Não fuma e ingere álcool com moderação & $13 \%$ & $14 \%$ & $4 \%$ & $69 \%$ \\
\hline & $\begin{array}{l}\text { Sempre utiliza cinto de segurança, respeita as normas de transito, nunca } \\
\text { ingerindo álcool se vai dirigir }\end{array}$ & $5 \%$ & $5 \%$ & $15 \%$ & $75 \%$ \\
\hline \multirow{3}{*}{ 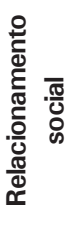 } & Procura cultivar amigos e está satisfeito com seus relacionamentos & $0 \%$ & $4 \%$ & $27 \%$ & $69 \%$ \\
\hline & $\begin{array}{l}\text { No lazer inclui reuniões com os amigos, atividades esportivas em grupos, } \\
\text { participação em associações }\end{array}$ & $4 \%$ & $17 \%$ & $42 \%$ & $37 \%$ \\
\hline & Procura ser ativo na comunidade, sentindo-se útil no ambiente social & $6 \%$ & $19 \%$ & $44 \%$ & $31 \%$ \\
\hline \multirow{3}{*}{ 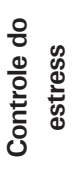 } & Reserva tempo (ao menos 5 minutos) todos os dias para relaxar & $3 \%$ & $18 \%$ & $27 \%$ & $52 \%$ \\
\hline & Mantém uma discussão sem alterar-se, mesmo quando contrariado & $7 \%$ & $26 \%$ & $46 \%$ & $21 \%$ \\
\hline & Equilibra o tempo dedicado ao trabalho com o tempo dedicado ao lazer & $4 \%$ & $16 \%$ & $45 \%$ & $35 \%$ \\
\hline
\end{tabular}


realizam atividades físicas por mais de 30 minutos durante cinco dias da semana; b) no segundo item vemos que $2 \%$ apresentaram índice negativo e $18 \%$ índice regular, porém, os $80 \%$ restantes apresentaram índices positivos por afirmarem que sempre ou quase sempre, no seu dia a dia, realizam exercícios que envolvam força e alongamento muscular; c) o terceiro item apresentou $6 \%$ de índice negativo, 30\% de índice regular e os demais obtiveram índices positivos, por responderem que sempre ou quase sempre, vão ao trabalho andando ou pedalando, realizando assim uma determinada atividade física, conforme preconiza este estudo.

A atividade física, em um sentido mais restrito, é todo movimento corporal, produzido por músculos esqueléticos, que provoca um gasto de energia. ${ }^{9}$ As atividades físicas realizadas adequadamente podem ter efeitos positivos na saúde, inclusive prolongando a vida $^{12}$. Exercícios de força podem influenciar nas modificações da composição corporal, redução da ansiedade, depressão além da melhora cardiovascular. ${ }^{13}$

Neste estudo foram apontados vários benefícios da atividade física, então se faz mais do que necessário desenvolver estas atividades em nosso cotidiano, para assim conseguimos mantermos nossa saúde em boa situação.

No componente comportamento preventivo foi observado o que segue: a) no primeiro item podemos observar que $13 \%$ dos acadêmicos não conhecem seus níveis de pressão arterial e de colesterol, ficando assim com índice negativo. Outros (27\%) dos entrevistados ficaram com índice regular, pois raramente conhecem seus níveis de pressão arterial e de colesterol e os demais estudantes apresentaram índices positivos, pois afirmaram que sempre ou quase sempre, conhecem seus níveis de pressão arterial e de colesterol. O colesterol total tem seu melhor nível, quando está abaixo de 200, acima disso pode ocasionar riscos para um ataque do coração; ${ }^{14}$ b) no segundo item, 13\% apresentam índice negativo e 14\% apresentam índice regular; já os estudantes restantes apresentaram índice positivo, pois informaram que sempre ou quase sempre não fazem uso de tabaco, nem de álcool ou se fazem uso de álcool é com moderação (menos de duas doses ao dia). Segundo Lopes, ${ }^{3}$ no Brasil o cigarro leva ao óbito de cerca de 200 mil todos os anos, logo o não uso desta substância evitará os danos por ela causados; c) no terceiro item, 90\% dos entrevistados apresentaram índice positivo, pois sempre ou quase sempre, fazem uso do cinto de segurança, não ingerem bebidas alcoólicas antes de dirigir e respeitam as normas de trânsito em seu cotidiano, enquanto $(5 \%)$ dos acadêmicos tiveram índice regular e 5\% apresentaram índice negativo, pois raramente ou não, respectivamente, respeitam as normas de trânsito.

No componente relacionamento social foi destacado o seguinte: a) no primeiro item, dos entrevistados, $69 \%$ apresentaram índice positivo, pois afirmaram que sempre ou quase sempre, estão satisfeitos com seus amigos e procuram mantê-los, enquanto $4 \%$ dos acadêmicos apresentaram índice regular, pois raramente estão satisfeitos e cultivam suas amizades, bem como nenhum dos alunos apresentaram índice negativo; b) no segundo item, $4 \%$ dos alunos tiveram índice negativo, pois informaram que, em suas rotinas, não incluem em suas rotinas diárias encontro com amigos e atividades esportivas em grupo, outros $17 \%$ dos entrevistados afirmaram que raramente incluem encontro com amigos e atividades esportivas em grupo em seu cotidiano, apresentando assim índice regular, enquanto os outros entrevistados apresentaram índice positivo, por afirmarem que sempre ou quase sempre, incluem encontro com amigos e atividades esportivas em grupo em suas rotinas diárias; c) no terceiro item, podemos observar que dos acadêmicos $6 \%$ apresentaram índice negativo, enquanto $19 \%$ apresentaram índice regular, por não ou raramente, respectivamente, serem ativos em suas comunidades, enquanto os outros entrevistados apresentaram índice positivo, pois em suas comunidades participam ativamente e se sentem úteis à sua comunidade.

A formação do homem se dá a partir da interação com o meio no qual se encontra inserido. ${ }^{15}$ Logo passamos a ver o quanto as relações sociais estão presentes e são importantes para nossa vida, então é essencial que tenhamos índices positivos neste componente para que tenhamos um estilo de vida que venha a trazer benefícios à saúde.

No componente controle do stress, desenharam-se os seguintes resultados: a) no primeiro item, 3\% dos acadêmicos apresentaram índice negativo e $18 \%$ índice regular, por respectivamente não e raramente, tirarem 5 minutos para relaxarem diariamente, enquanto os outros alunos apresentaram índice positivo, por afirmarem que sempre ou quase sempre, tiram 5 minutos para relaxarem diariamente; b) no segundo item, 7\% dos entrevistados tiveram índice negativo, enquanto $26 \%$ tiveram índice regular, por não ou raramente respectivamente, se alterarem em uma discussão quando contrariados e os demais acadêmicos apresentaram índice positivo, pois declararam que não se alteram em uma discussão, quando contrariados; c) no terceiro item, podemos observar que $4 \%$ e $16 \%$ dos acadêmicos, apresentaram índice negativo e regular respectivamente, por não e raramente, dividirem tempo de trabalho com lazer, enquanto os outros alunos, por afirmarem que sempre ou quase sempre, dividem o tempo de trabalho com lazer em seu cotidiano, apresentaram índice positivo.

Estes três últimos componentes são responsáveis diretos do controle ao estresse e garante o bem-estar de quem vive, adotando estes itens positivamente em seus estilos de vida, pois o estresse estando fora de controle traz um risco imensurável a saúde. ${ }^{8}$

Os principais componentes que integram o comportamento preventivo com relação à saúde são enfatizados pelos autores do O Pentáculo do Bem-Estar. ${ }^{8}$ Com isso entendemos que as ações que realizamos de forma cautelar, ou de segurança, no intuito de evitar danos à vida, ou prejuízos à saúde são os comportamentos preventivos e junto deste entendimento, já vemos a importância que este componente tem no estilo de vida de uma pessoa, pois é através dele que desenvolvemos atitudes que vão prevenir os riscos à nossa saúde, ou a nossa própria vida.

\section{CONSIDERACÕES FINAIS}

Pôde ser observada uma positividade bastante presente em todos os componentes pesquisados que, em apenas alguns itens, apresentaram índices de estilo de vida regular e que em nenhum deles, prevaleceu 
índice negativo.

Portanto, podemos concluir que o perfil de estilo de vida do grupo estudado nesta pesquisa é considerado positivo de acordo com a literatura, por prevalecer um maior índice positivo nas afirmações encontradas em cada componente, porém, por terem apresentado índice regular em alguns poucos itens, sugere-se ao público alvo que adote meios para estarem sempre em busca do índice positivo, bem como, a Instituição onde foi realizada a pesquisa, a implementação de ações de conscientização e de incentivo para uma melhora do estilo de vida de seus acadêmicos de forma geral.

\section{REFERÊNCIAS}

1. World Health Organization. Milestones in Health Promotion: Statements from Global Conferences. Retrievedfrom 2009. OMS.

2. Minayo MCS, Hartz ZMA, Buss PM. Qualidade de vida e saúde: um debate necessário. Ciência \& Saúde Coletiva 2000;5(1):7-18.

3. Lopes JL. Análise do perfil de estilo de vida dos servidores da Prefeitura Municipal de Astorga. Paraná: Maringá, 2009.

4. Pozena R, Cunha NFS. Saúde e Sociedade. São Paulo: Lourdes, 2009.

5. Souza DLG. O mito do sedentarismo. EFDeportes.com, Revista Digital 2010;15(151):1-1.
6. Santos DH. A influência do estilo de vida na formação do adolescente. 2014. 36 f. Trabalho de Conclusão de Curso (Especialização) Universidade Tecnológica Federal do Paraná, Medianeira, 2014.

7. Gonçalves A, Vilarta R. Qualidade de vida e atividade física: explorando teoria e prática. Manole, 2004.

8. Nahas MV, Barros MVG, Francalacci VL. O Pentáculo do bem-estar: base conceitual para avaliação do estilo de vida de indivíduos ou grupos. Revista Brasileira de Atividade Física e Saúde 2000;5(2):48-59.

9. Barbanti VJ. Dicionário de Educação Física e do Esporte. Manole, 2003

10. Nahas MV. Atividade física, saúde e qualidade de vida: conceitos e sugestões para um estilo de vida ativo. 5. ed. rev. atual. Londrina: Midiograf, 2010. 318p.

11. Pinheiro ARO. A alimentação saudável e a promoção da saúde no contexto da segurança alimentar e nutricional. Saúde em Debate 2005;29(70):125-139.

12. Nahas MV. Atividade física, saúde e qualidade de vida: conceitos e sugestões para um estilo de vida ativo. Midiograf 2003, 3. ed.

13. Simão R. Treinamento de força na saúde e na qualidade de vida. Phorte, 2004.

14. Guyton AC, Hall JE. Tratado de fisiologia médica. Elsevier 2011, 12. ed.

15. Oliveira MK. Vygotsky: Aprendizado e desenvolvimento: um processo sócio histórico. São Paulo: Scipione, 1993. 